\title{
Ordinal Analysis and the Infinite Ramsey Theorem
}

\author{
Bahareh Afshari and Michael Rathjen
}

\begin{abstract}
The infinite Ramsey theorem is known to be equivalent to the statement 'for every set $X$ and natural number $n$, the $n$-th Turing jump of $X$ exists, over $\mathrm{RCA}_{0}$ due to results of Jockusch [5]. By subjecting the theory $\mathrm{RCA}_{0}$ augmented by the latter statement to an ordinal analysis, we give a direct proof of the fact that the infinite Ramsey theorem has proof-theoretic strength $\varepsilon_{\omega}$. The upper bound is obtained by means of cut elimination and the lower bound by extending the standard well-ordering proofs for $\mathrm{ACA}_{0}$. There is a proof of this result due to McAloon [6], using model-theoretic and combinatorial techniques. According to [6], another proof appeared in an unpublished paper by Jäger.
\end{abstract}

\section{Introduction}

Ramsey's theorem for infinite sets asserts that for every $k \geq 1$ and colouring of the $k$-element subsets of $\mathbb{N}$ with finitely many colours, there exists an infinite subset of $\mathbb{N}$ all of whose $k$-element subsets have the same colour [7]. We will denote the previous statement, when specialised to a fixed $k$, by $\mathrm{RT}(k)$. It is well known that $\mathrm{ACA}_{0}$ is equivalent to $\mathrm{RT}(k)$ for any (outer world) $k \geq 3$ [11]. However, the general assertion of Ramsey's theorem, $\forall x \mathrm{RT}(x)$ (abbreviated henceforth by $\mathrm{iRT}$ ), is stronger than $\mathrm{ACA}_{0}$.

For $b \geq 1$ we write $F:[A]^{n} \rightarrow b$ to signify that $F$ maps the $n$-element subsets of $A$ into the set $\{0, \ldots, b-1\} . X \subseteq A$ is said to be monochromatic for $F$ if $F$ is constant on $[X]^{n}$.

It is known from work of Jockusch [5, Theorem 5.7] that iRT is not provable in $\mathrm{ACA}_{0}$. More precisely, for every $n \geq 0$, there is a recursive $F:[\mathbb{N}]^{n+2} \rightarrow 2$ such that the $n$-th Turing jump of $\emptyset$ is recursive in any infinite $F$-monochromatic $X \subseteq \mathbb{N}$. On the other hand, it also follows from [5, Theorem 5.6] that for every recursive $F:[\mathbb{N}]^{n} \rightarrow b$ and $n \geq 0$ there exists an $F$-monochromatic $X$ recursive in the $n$-th Turing jump of $\emptyset$.

For $X, Y \subseteq \mathbb{N}$ and $n<\omega$, let $j u m p(n, X, Y)$ abbreviate the formula stating that $Y$ is the $n$-th Turing jump of $X$. By relativising the results of [5], we arrive at the following theorem (cf. [6]).

Theorem 1. $\mathrm{ACA}_{0}+\forall n \forall X \exists Y j u m p(n, X, Y)$ and $\mathrm{ACA}_{0}+\mathrm{iRT}$ prove the same statements. 
By [6], $\mathrm{ACA}_{0}+\mathrm{iRT}$ has the same first order consequences as the theory obtained from PA by iterating the uniform reflection principle arbitrarily often. This will also follow from Theorem 21, in light of the well-known fact that the latter theory has proof-theoretic ordinal $\varepsilon_{\omega}$ (see [8]). It is also worth mentioning that the paper [12] (whose results actually postdate those in the present paper) contains, among other things, a characterization of the $\Pi_{2}^{0}$ consequences of $\mathrm{ACA}_{0}$ augmented by the infinite Ramsey theorem.

We fix a primitive recursive ordering $\preceq$ on $\omega$ of order type $\Gamma_{0}$. For $\alpha \preceq \Gamma_{0}$, let $\operatorname{TI}(\prec \alpha)$ denote the scheme of transfinite induction on initial segments of $\alpha$, i.e.

$$
\forall \xi(\forall \delta \prec \xi A(\delta) \rightarrow A(\xi)) \rightarrow \forall \xi \prec \bar{\beta} A(\xi),
$$

for every $\beta \prec \alpha$ and every arithmetical formula $A(x)$. Here $\bar{\beta}$ denotes the numeral corresponding to the ordinal $\beta$. The proof-theoretic ordinal of a theory $T$, is the least $\alpha$ such that $T$ is equivalent to $\mathrm{PA}+\mathrm{TI}(\prec \alpha)$, in the sense that they prove the same statements of arithmetic, and this fact can be established on the basis of PA.

The theory $\mathrm{ACA}_{0}+\forall n \forall X \exists Y j u m p(n, X, Y)$, commonly denoted by $\mathrm{ACA}_{0}^{\prime}$, has previously been shown to have proof-theoretic ordinal $\varepsilon_{\omega}$ in [6]. The latter paper uses model-theoretic and combinatorial techniques but also draws on proof-theoretic results in order to construct an instance of transfinite induction up to $\varepsilon_{\omega}$ that cannot be proven in $\mathrm{ACA}_{0}^{\prime}$, and thereby indicates that the strength of the theory is bounded by $\varepsilon_{\omega}$. An unpublished proof using prooftheoretic means is attributed to Jäger [6]. However, to the authors' knowledge no proof using cut elimination is available in the published literature. This paper provides a simple proof-theoretic ordinal analysis of the system $\mathrm{ACA}_{0}+\forall n \forall X \exists Y j u m p(n, X, Y)$. The upper bound is obtained by means of cut elimination and the lower bound by extending the standard well-ordering proofs for $\mathrm{ACA}_{0}$ as in [9]. For the definitions of systems of comprehension, ordinal notation and other basic definitions we refer the reader to $[1,11]$. The results presented here form part of [1].

\section{The semi-formal system $\mathrm{ACA}_{\infty}$}

Let $\mathrm{ACA}_{\infty}$ be the infinitary calculus corresponding to $\mathrm{ACA}_{0}$. Informally, the system $\mathrm{ACA}_{\infty}$ is obtained from $\mathrm{ACA}_{0}$ by replacing the set induction axiom, i.e. $\forall X(0 \in X \wedge \forall x(x \in X \rightarrow x+1 \in X) \rightarrow \forall n n \in X)$, by the infinitary $\omega$-rule.

The language of $\mathrm{ACA}_{\infty}$ is the same as that of $\mathrm{ACA}_{0}$ but the notion of a formula comes enriched with predicators. Formulae and predicators are defined simultaneously. Literals (atomic or negated atomic formulae) are formulae. Every set variable is a predicator. If $A(x)$ is a formula without set quantifiers, i.e. arithmetical, then $\{x \mid A(x)\}$ is a predicator. If $P$ is a predicator and $t$ is a numerical term, then $t \in P$ and $t \notin P$ are formulae. The other formation rules pertaining to $\wedge, \vee, \forall x, \exists x, \forall X, \exists X$ are as usual. 
We will be working in a Tait-style formalisation of the second order arithmetic. By a sequent $\Gamma$ we mean a finite set of formulae in the language of second order arithmetic, $\mathcal{L}_{2}$. Due to the presence of the $\omega$-rule we need only consider formulae without free numerical variables.

The axioms of the system $\mathrm{ACA}_{\infty}$ are

- $\Gamma, L$ where $L$ is a true literal;

- $\Gamma, s \in X, t \notin X$ where $s$ and $t$ are terms of the same value.

The rules of $\mathrm{ACA}_{\infty}$ are

$$
\begin{array}{cc}
\left(\vee_{i}\right) \frac{\Gamma, A_{i} \text { for } i<2}{\Gamma, A_{0} \vee A_{1}} & (\wedge) \frac{\Gamma, A_{0} \quad \Gamma, A_{1}}{\Gamma, A_{0} \wedge A_{1}} \\
(\omega) \frac{\Gamma, A(\bar{n}) \text { for all } n}{\Gamma, \forall x A(x)} & \left(\exists_{1}\right) \frac{\Gamma, A(s)}{\Gamma, \exists x A(x)} \\
\left(\forall_{2}\right) \frac{\Gamma, A(X) X \text { not free in } \Gamma}{\Gamma, \forall X A(X)} & \left(\exists_{2}\right) \frac{\Gamma, A\left(\left\{x \mid A_{0}(x)\right\}\right)}{\Gamma, \exists X A(X)} \\
\left(\operatorname{Pr}_{1}\right) \frac{\Gamma, A(t)}{\Gamma, t \in\{x \mid A(x)\}} & \left(\operatorname{Pr}_{2}\right) \frac{\Gamma, \neg A(t)}{\Gamma, t \notin\{x \mid A(x)\}} \\
(\mathrm{Cut}) \frac{\Gamma, A \quad \Gamma, \neg A}{\Gamma} &
\end{array}
$$

The rank of a formula $A$, denoted $|A|$, is defined as follows.

- $|L|=0$, if $L$ is a literal.

- $|s \in P|=|s \notin P|=|A(\overline{0})|+1$, if $P$ is the predicator $\{x \mid A(x)\}$.

- $\left|A_{0} \wedge A_{1}\right|=\left|A_{0} \vee A_{1}\right|=\max \left\{\left|A_{0}\right|,\left|A_{1}\right|\right\}+1$.

- $|\forall x A(x)|=|\exists x A(x)|=|A(x)|+1$.

- $|\forall X A(X)|=|\exists X A(X)|=\max \{|A(X)|+1, \omega\}$.

For ordinals $\alpha, \kappa \prec \Gamma_{0}$, we write $\operatorname{ACA}_{\infty} \mid \frac{\alpha}{\kappa} \Gamma$ to convey that the sequent $\Gamma$ is deducible in $\mathrm{ACA}_{\infty}$ via a derivation of length $\preceq \alpha$ containing only cuts on formulae of rank $\prec \kappa$. More precisely, this notion is defined inductively as follows: If $\Gamma$ is an axiom of $\mathrm{ACA}_{\infty}$ then $\mathrm{ACA}_{\infty} \mid \frac{\alpha}{\kappa} \Gamma$ holds for any $\alpha$ and $\kappa$. If $\alpha_{i}<\alpha$ and $\mathrm{ACA}_{\infty} \mid \frac{\alpha_{i}}{\kappa} \Gamma_{i}$ hold for all premisses $\Gamma_{i}$ of an $\mathrm{ACA}_{\infty}$-inference with conclusion $\Gamma$, then $\mathrm{ACA}_{\infty} \mid \frac{\alpha}{\kappa} \Gamma$, provided that in the case of (Cut) the cutformulae also have ranks $\prec \kappa$.

$\mathrm{ACA}_{\infty}$ corresponds to the system $\mathrm{EA}^{*}$ in [9] and can be interpreted into the first level of the semi-formal system of Ramified analysis, $\mathrm{RA}^{*}$. The fact that $\mathrm{ACA}_{\infty}$ 
enjoys cut elimination is folklore and the proof involves the standard techniques of predicative proof theory. For proofs of the following see, for example, [1, 9].

\section{Lemma 2.}

1. $\mathrm{ACA}_{\infty} \mid \frac{|A| \cdot 2}{0} \Gamma, A, \neg A$ for every arithmetical formula $A$.

2. If $\Gamma$ contains an axiom of $\mathrm{ACA}_{0}$, then $\mathrm{ACA}_{\infty} \mid \frac{\omega+k}{0} \Gamma$ for some $k<\omega$.

Theorem 3 (First Cut Elimination Theorem for $\mathrm{ACA}_{\infty}$ ). Let $\alpha, \beta \prec \Gamma_{0}$ and $k<\omega$. If $\mathrm{ACA}_{\infty} \mid \frac{\alpha}{\beta+k} \Gamma$, then $\mathrm{ACA}_{\infty} \mid \frac{\omega_{k}(\alpha)}{\beta} \Gamma$ where $\omega_{0}(\alpha)=\alpha$ and $\omega_{k+1}(\alpha)$ $=\omega^{\omega_{k}(\alpha)}$.

Theorem 4 (Second Cut Elimination Theorem for $\mathrm{ACA}_{\infty}$ ). Let $\omega \preceq \alpha \prec \Gamma_{0}$. If $\mathrm{ACA}_{\infty} \mid \frac{\alpha}{\omega} \Gamma$, then $\mathrm{ACA}_{\infty} \mid \varepsilon_{0} \frac{\varepsilon^{\prime}}{\Gamma}$.

\section{An upper bound for $\mathrm{ACA}_{0}+\mathrm{iRT}$}

Let $T$ denote the theory $\mathrm{ACA}_{0}+\forall n \forall X \exists Y j u m p(n, X, Y)$. We will obtain an upper bound on the strength of this theory by a combination of embeddings and cut elimination theorems. We first embed $T$ into an intermediate theory $T^{*}$. The semi-formal system $T^{*}$ has the same language as $\mathrm{ACA}_{\infty}$. If $A$ is a formula of $T$, then we write $A^{*}$ to refer to any formula obtained from $A$ by substituting all number variables by arbitrary closed terms. The system $T^{*}$ has as axioms all sequences $\Gamma, A^{*}$ such that $A$ is a basic axiom of $\mathrm{ACA}_{0}$ or the set induction axiom. Moreover, we have the following axioms in $T^{*}$.

- $\Gamma, A, \neg A$ if $A$ is arithmetical.

- $\Gamma, j u m p\left(\bar{n}, P, S_{n}^{P}\right)$ for every $n$ and arithmetical predicator $P$.

In above, $S_{n}^{P}$ is the arithmetical predicator which defines the $n$-th Turing jump of $P$. The predicator $S_{n}^{P}$ is formally defined as follows.

$$
\begin{aligned}
S_{0}^{P} & =\{x \mid \exists u[x=\langle 0, u\rangle \wedge u \in X]\}, \\
S_{n+1}^{P} & =\left\{x \mid \exists u\left[x=\langle\overline{n+1}, u\rangle \wedge \exists v\{u\}^{\left(S_{n}^{P}\right)_{n}}(u)=v\right]\right\},
\end{aligned}
$$

where $\langle.,$.$\rangle is a primitive recursive pairing function, \{u\}^{Y}$ represents the $u$-th partial recursive function with oracle $Y$, and $(Y)_{n}$ denotes the $n$-slice of the set $Y$, i.e. $(Y)_{n}=\{y \mid\langle n, y\rangle \in Y\}$. The logical rules of $T^{*}$ are the same as in $\mathrm{ACA}_{\infty}$. The rank of a formula and notation $\left.T^{*}\right|_{\kappa} ^{\alpha} \Gamma$ are defined analogously. From the choice of the axioms of $T^{*}$ and the fact that the rank of a formula is always strictly less than $\omega+\omega$ we can derive the following.

Theorem 5 (Embedding Theorem). Suppose $T \vdash B$. Then there exist natural numbers $n$ and $m$ such that $T^{*} \frac{n}{\omega+m} B^{*}$ holds for all $B^{*}$. 
We now perform partial cut elimination in $T^{*}$. The following reduction lemma goes through in the standard way.

Lemma 6 (Reduction Lemma). Let $n_{0}, n_{1}<\omega$ and $|A|=\kappa \succeq \omega$. If $T^{*} \frac{n_{0}}{\kappa} \Gamma, A$ and $T^{*} \mid \frac{n_{1}}{\kappa} \Delta, \neg A$, then $T^{*} \mid \frac{n_{0}+n_{1}}{\kappa} \Gamma, \Delta$.

Proof. By induction on the sum $n_{0}+n_{1}$. We show the interesting case where both $A$ and $\neg A$ are active in the derivations and $A$ is derived via the $\left(\forall_{2}\right)$-rule. Suppose $A$ is of the form $\forall Y A_{0}(Y)$ and we have

$$
\frac{(1) \mid \frac{m_{0}}{\kappa} \Gamma, A_{0}(X),\left[\forall Y A_{0}(Y)\right] \quad m_{0}<n_{0}}{\left.(2) \quad\right|_{\kappa} ^{n_{0}} \Gamma, \forall Y A_{0}(Y)}\left(\forall_{2}\right)
$$

where $X$ is not free in $\Gamma \cup\left\{\forall Y A_{0}(Y)\right\}$, and

$$
\frac{(3) \mid \frac{m_{1}}{\kappa} \Delta, \neg A_{0}(P),\left[\exists Y \neg A_{0}(Y)\right] \quad m_{1}<n_{1}}{(4) \quad \frac{n_{1}}{\kappa} \Delta, \exists Y \neg A_{0}(Y)}\left(\exists_{2}\right)
$$

where $P$ is an arithmetical predicator. In the above inferences, we write $[B]$ to emphasise that the formula $B$ may or may not have appeared in the premise of the original inference. Applying the induction hypothesis to (2) and (3) yields

$$
\mid \frac{n_{0}+m_{1}}{\kappa} \Gamma, \Delta, \neg A_{0}(P),
$$

and to (1) and (4) yields

$$
\mid \frac{m_{0}+n_{1}}{\kappa} \Gamma, \Delta, A_{0}(X)
$$

It is not hard to show that (6) implies $\frac{m_{0}+n_{1}}{\kappa} \Gamma, \Delta, A_{0}(Q)$ for any arithmetical predicator $Q$ and in particular

$$
\frac{m_{0}+n_{1}}{\kappa} \Gamma, \Delta, A_{0}(P) .
$$

Since $\left|A_{0}(P)\right| \prec\left|\forall Y A_{0}(Y)\right|=\kappa$, we may perform a cut on (5) and (7) to conclude $\frac{n_{0}+n_{1}}{\kappa} \Gamma, \Delta$ as required. For full details see $[1, \S 3]$.

Theorem 7 (Cut Elimination Theorem). If $\left.T^{*}\right|_{\omega+m+1} ^{n} \Gamma$ for some $m, n<\omega$, then $T^{*} \frac{2^{2^{n}}}{\omega+m} \Gamma$.

Proof. By induction on $n$. If $\Gamma$ is an axiom, then $\vdash_{0}^{0} \Gamma$. Otherwise, there are two cases to consider. Suppose $\Gamma$ is of the form $\Gamma^{\prime}, A$ and we have

$$
\frac{\left.\right|_{\omega+m+1} ^{n_{i}} \Gamma^{\prime}, A_{i} \quad i<\omega, n_{i}<n}{\frac{n}{\omega+m+1} \Gamma^{\prime}, A}(R)
$$


where $R$ is any of the rules of $T^{*}$ except the cut rule. By applying the induction hypothesis to the premise(s) of the above inference we obtain $\frac{2^{n_{i}}}{\omega+m} \Gamma^{\prime}, A_{i}$. Reapplying the rule $(R)$ yields $\frac{2^{n}}{\omega+m} \Gamma$.

If the last inference was a cut, namely,

$$
\frac{\frac{n_{0}}{\omega+m+1} \Gamma, A \quad \frac{n_{1}}{\omega+m+1} \Gamma, \neg A}{\frac{n}{\omega+m+1}^{n} \Gamma}(\mathrm{Cut})
$$

where $n_{0}, n_{1}<n$ and $|A| \preceq \omega+m$, by applying the induction hypothesis to the premises of the above cut we obtain

$$
\left.\right|_{\omega+m} ^{2^{n_{0}}} \Gamma, A \text { and }\left.\right|_{\omega+m} ^{2^{n_{1}}} \Gamma, \neg A .
$$

If $|A| \prec \omega+m$, by a cut on $A$ we derive $\frac{2^{n}}{\omega+m} \Gamma$. Otherwise, the Reduction Lemma yields $\frac{2^{n_{0}}+2^{n_{1}}}{\omega+m} \Gamma$, thus by monotonicity we obtain the desired result.

Corollary 8. For $n, m<\omega$, if $\left.T^{*}\right|_{\omega+m} ^{n} \Gamma$, then $\left.T^{*}\right|_{\omega} ^{2_{m}^{n}} \Gamma$ where $2_{0}^{n}=n$ and $2_{k+1}^{n}=2^{2_{k}^{n}}$.

Finally, to analyse $T$ we will embed $T^{*}$ into $\mathrm{ACA}_{\infty}$ so that we can eliminate the remaining cuts and read off an upper bound. First we need the following lemma, which can be verified by induction.

Lemma 9. There are primitive recursive functions $f, g$ such that for each $n$, $f(n), g(n)<\omega$ and $\mathrm{ACA}_{\infty} \frac{f(n)}{g(n)} \Gamma, j u m p\left(\bar{n}, P, S_{n}^{P}\right)$.

Theorem 10. Let $\Gamma$ be a finite set of arithmetical formulae and $k<\omega$. Then $T^{*} \mid \frac{k}{\omega} \Gamma$ implies $\mathrm{ACA}_{\infty} \mid \frac{\varepsilon_{k}}{0} \Gamma$.

Proof. By induction on $k$. If $\Gamma$ is an axiom of $T^{*}$ we have the following three cases to consider. If $\Gamma$ is the sequent $\Gamma^{\prime}, A^{*}$ where $A$ is a basic axiom of $\mathrm{ACA}_{0}$ or the set induction axiom, then we have, by Lemma 2 , that $\mathrm{ACA}_{\infty} \mid \frac{\omega+k}{0} \Gamma^{\prime}, A^{*}$ for some $k<\omega$. If $\Gamma$ is the sequent $\Gamma^{\prime}, \operatorname{jump}\left(\bar{n}, P, S_{n}^{P}\right)$ where $P$ is an arithmetical predicator, Lemma 9 yields $\mathrm{ACA}_{\infty} \mid \frac{f(n)}{g(n)} \Gamma, j u m p\left(\bar{n}, P, S_{n}^{P}\right)$. By applying the First Cut Elimination theorem for $\mathrm{ACA}_{\infty}$ we obtain

$$
\mathrm{ACA}_{\infty} \mid \frac{\omega_{g(n)}(f(n))}{0} \Gamma, j u m p\left(\bar{n}, P, S_{n}^{P}\right),
$$

with $\omega_{k}$ defined as in Theorem 3. Since $f(n), g(n)<\omega$, we have $\omega_{g(n)}(f(n)) \prec$ $\varepsilon_{0}$, and hence may deduce $\mathrm{ACA}_{\infty} \mid \frac{\varepsilon_{0}}{0} \Gamma, \operatorname{jump}\left(\bar{n}, P, S_{n}^{P}\right)$. Let $\Gamma$ be of the form $\Gamma^{\prime}, A, \neg A$ with $A$ being arithmetical. As $\mathrm{ACA}_{\infty} \mid \frac{|A| \cdot 2}{0} \Gamma, A, \neg A$ holds due to Lemma 2, monotonicity provides the desired result.

Now suppose $\Gamma$ is derived by an application of a logical rule in $T^{*}$. If the last inference is a cut, then we have 


$$
\frac{T^{*} \frac{k_{0}}{\omega} \Gamma, A \quad T^{*} \mid \frac{k_{0}}{\omega} \Gamma, \neg A}{T^{*} \mid \frac{k}{\omega} \Gamma}(\mathrm{Cut})
$$

where $k_{0}<k$ and $A$ is arithmetical. Applying the induction hypothesis to the premises of the above cut yields $\mathrm{ACA}_{\infty} \frac{\varepsilon_{k_{0}}}{0} \Gamma, A$ and $\left.\mathrm{ACA}_{\infty}\right|_{0} ^{\varepsilon_{k_{0}}} \Gamma, \neg A$. Applying a cut to $A$, we conclude that $\mathrm{ACA}_{\infty} \mid \frac{\varepsilon_{k_{0}}+1}{m} \Gamma$ for $m=|A|+1$. Thus $\mathrm{ACA}_{\infty} \mid \frac{\omega_{m}\left(\varepsilon_{k_{0}}+1\right)}{0} \Gamma$ and so we may deduce $\mathrm{ACA}_{\infty} \mid \frac{\varepsilon_{k}}{0} \Gamma$. have

If $\Gamma$ is derived via the $\left(\forall_{2}\right)$-rule, $\Gamma$ must be of the form $\Gamma^{\prime}, \forall Y A_{0}(Y)$ and we

$$
\frac{T^{*} \mid \frac{k_{0}}{\omega} \Gamma^{\prime}, A_{0}(X) \quad X \text { is not free in } \Gamma^{\prime} \text { and } k_{0}<k}{T^{*} \mid \frac{k}{\omega} \Gamma^{\prime}, \forall Y A_{0}(Y)}\left(\forall_{2}\right)
$$

Applying the induction hypothesis to the premise of the above inference yields $\mathrm{ACA}_{\infty} \mid \frac{\varepsilon_{k_{0}}}{0} \Gamma^{\prime}, A_{0}(X)$. Re-applying $\left(\forall_{2}\right)$-rule allows us to deduce $\mathrm{ACA}_{\infty} \mid \frac{\varepsilon_{k}}{0} \Gamma$. The other cases are similar.

Corollary 11. Every arithmetical theorem of $T$ without set variables is derivable in $\mathrm{PA}+\mathrm{TI}\left(\prec \varepsilon_{\omega}\right)$.

Proof. Suppose $A$ is an arithmetical sentence and $T \vdash A$. By the Embedding Theorem $T^{*} \frac{n}{\omega+m} A$ holds for some $n, m<\omega$. Cut elimination for $T^{*}$ yields $\left.T^{*}\right|_{\omega} ^{k} A$ holds for some $k<\omega$. By embedding $T^{*}$ into $\mathrm{ACA}_{\infty}$ we arrive at $\mathrm{ACA}_{\infty}||_{0}^{\varepsilon_{k}} A$. This means that $A$ is derivable in $\mathrm{ACA}_{\infty}$ directly from the axioms and first order rules, and, moreover, if $A$ is of complexity $\Pi_{n}^{0}$ for some $n$, then all formulae occurring in this cut-free derivation belong to the same complexity class. By employing a partial truth predicate for $\Pi_{n}^{0}$-formulae and transfinite induction up to $\varepsilon_{k+1}$, one shows that $\mathrm{PA}+\operatorname{TI}\left(\prec \varepsilon_{\omega}\right) \vdash A$ (cf. [10]).

\section{A lower bound for $\mathrm{ACA}_{0}+\mathrm{iRT}$}

To deduce that $\varepsilon_{\omega}$ is also a lower bound for the strength of the theory $T$, we will show that $T$ can prove the well-foundedness of all ordinals strictly less than $\varepsilon_{\omega}$. Our method is to extend the standard well-ordering proofs for $\mathrm{ACA}_{0}$ as for instance given in [9, Theorem 23.3]. Let us denote by $S p$ the operator defined by

$$
S p(V)=\left\{\alpha \mid \forall \xi\left(\xi \subset V \rightarrow \xi+\omega^{\alpha} \subset V\right)\right\},
$$

where $\xi \subset V$ abbreviates $\forall x(x \prec \xi \rightarrow x \in V)$. For sets $X$ and $Y$ we write $X \leq_{e}$ $Y$ to convey that $\forall x\left(K_{X}(x)=\{e\}^{Y}(x)\right)$, where $K_{X}$ denotes the characteristic function of the set $X$. The aim of the next few lemmata is to establish that in the theory $T$ finite iterations of the $S p$ operator can be coded into a single set. They are easy to verify using the Kleene $T$-predicate and $S$ - $m$ - $n$ theorem [3]. Detailed proofs can be found in $[1, \S 3]$. In the following $X^{(n)}$ denotes the $n$-th 
Turing jump of $X$, i.e. the set $Y$ such that $j u m p(n, X, Y)$. We also use $X^{\prime}$ and $X^{\prime \prime}$ respectively for $X^{(1)}$ and $X^{(2)}$.

Lemma 12. Let $A(x, y, z, U)$ be a $\Delta_{0}^{0}$-formula (of the language of $\mathrm{ACA}_{0}$ ) with all the free variables exhibited and $U$ being a free set variable. Then there exists a natural number e such that for every $X \subseteq \mathbb{N}$

$$
\{n \mid \forall x \exists y A(x, y, n, X)\} \leq_{e} X^{\prime \prime} .
$$

Proof. Since for every set $X \subseteq \mathbb{N}$ the set $\{\langle x, n\rangle: \exists y A(x, y, n, X)\}$ is recursively enumerable in $X$ (uniformly in $X$ ), there exists an index $e_{0}$ depending only on the formula $A(x, y, z, U)$ such that for all natural numbers $n$ and sets $X \subseteq \mathbb{N}$,

$$
\forall x \exists y A(x, y, n, X) \quad \text { iff } \quad \forall x\left\langle e_{0},\langle x, n\rangle\right\rangle \in X^{\prime} .
$$

Likewise, there is some $d_{0}$ such that for all sets $Y \subseteq \mathbb{N},\left\{d_{0}\right\}^{Y}$ is total and

$$
\forall x\left\langle e_{0},\langle x, n\rangle\right\rangle \in Y \quad \text { iff } \quad\left\langle d_{0},\left\langle e_{0}, n\right\rangle\right\rangle \in Y^{\prime} .
$$

It immediately follows from the $S-m-n$ theorem that there is an index $e$ such that $\{n \mid \forall x \exists y A(x, y, n, X)\} \leq_{e} X^{\prime \prime}$.

In particular, since $S p(X)$ is $\Pi_{2}^{0}$ in $X$ we can deduce the following.

Corollary 13. $\mathrm{ACA}_{0}$ proves the existence of a natural number e that satisfies $S p(X) \leq_{e} X^{\prime \prime}$ for all $X \subseteq \mathbb{N}$.

Lemma 14. There is a primitive recursive function $\circ: \omega \times \omega \rightarrow \omega$ such that for any sets $X, Y, Z$, if $X \leq_{e} Y$ and $Y \leq_{f} Z$, then $X \leq_{e o f} Z$.

Lemma 15. There is a primitive recursive function $N$ such that $X^{\prime} \leq_{N(e)} Y^{\prime}$ whenever $X \leq_{e} Y$.

Corollary 16. There exists a primitive recursive function $g$ such that

$$
S p^{n}(X) \leq_{g(n)} X^{(2 n)},
$$

where $S p^{n}$ is inductively defined as $S p^{0}(X)=X$ and $S p^{n+1}(X)=S p\left(S p^{n}(X)\right)$.

Proof. We define $g$ by induction on $n$. Suppose $n=0$. Let $g(0)$ be the index of the identity function. Then $S p^{0}(X) \leq_{g(0)} X$ holds trivially. For the induction step suppose $n=k+1$. By Corollary 13 there is an $e$ (independent of $k$ ) such that

$$
S p^{(k+1)}(X)=S p\left(S p^{(k)}(X)\right) \leq_{e}\left(S p^{(k)}(X)\right)^{(2)} .
$$

Using the induction hypothesis we may assume $S p^{(k)}(X) \leq_{g(k)} X^{(2 k)}$. Lemma 15 entails

$$
\left(S p^{(k)}(X)\right)^{(2)} \leq_{N(N(g(k)))}\left(X^{(2 k)}\right)^{(2)}=X^{(2 k+2)},
$$

and Lemma 14 yields

$$
S p^{k+1}(X) \leq_{g(k+1)} X^{(2 k+2)},
$$

by setting $g(k+1)=e \circ N(N(g(k)))$. Since $g$ is primitive recursive we are done. 
Lemma 17. $T \vdash \forall x \forall X \exists Y A(x, X, Y)$, where $A$ is the formula defined by $(Y)_{0}=$ $X \wedge \forall n<x(Y)_{n+1}=S p\left((Y)_{n}\right)$.

Proof. We argue informally within $T$. Given $x$ and $X$ define

$$
Y=\left\{\langle n, z\rangle \mid n \leq x \wedge\{g(n)\}^{X^{(2 n)}}(z) \simeq 0\right\},
$$

where $g$ is the primitive recursive function given by Corollary 16. It is easy to see that $(Y)_{0}=X$. Moreover, for $n \leq x$,

$$
z \in(Y)_{n} \quad \text { iff } \quad\{g(n)\}^{X^{(2 n)}}(z) \simeq 0 .
$$

By Corollary 16 we have $(Y)_{n}=S p^{(n)}(X)$. Thus $S p\left(S p^{(n)}(X)\right)=S p\left((Y)_{n}\right)$, and hence for $n<x$ we can deduce $(Y)_{n+1}=S p\left((Y)_{n}\right)$ as required.

Let $\operatorname{Tran}(\prec)$ and $\operatorname{LO}(\prec)$ be abbreviations for formulae stating $\prec$ is transitive and a linear order respectively. Fund $(\alpha, X)$ is the formula

$$
\operatorname{Tran}(\prec) \wedge\left(\operatorname{Prog}_{\prec}(X) \rightarrow \forall \xi \prec \alpha(\xi \in X)\right),
$$

where $\operatorname{Prog}_{\prec}(X)=\forall x(\forall y(y \prec x \rightarrow y \in X) \rightarrow x \in X)$, and $\operatorname{TI}(\alpha, X)$ is the formula

$$
\mathrm{LO}(\prec) \wedge \operatorname{Fund}(\alpha, X) .
$$

The following lemma is well known. For a proof see [9, 21 , Lemma 1].

Lemma 18. For every set $X$ and $\alpha \prec \Gamma_{0}$,

$$
\mathrm{ACA}_{0} \vdash \operatorname{Fund}(\alpha, S p(X)) \rightarrow \operatorname{Fund}\left(\omega^{\alpha}, X\right) .
$$

Lemma 19. $T \vdash$ Fund $\left(\varepsilon_{0}, X\right)$.

Proof. We argue informally within $T$. Fund $(\alpha, X)$ is progressive in $\alpha$, therefore it suffices to show $\forall n$ Fund $\left(\omega_{n}(\omega), X\right)$. By Lemma $17, A(n+1, X, Y)$ holds for some $Y$. On the other hand as induction up to $\omega$ is available in $T$ for every set, Fund $\left(\omega,(Y)_{n}\right)$ holds. Since $(Y)_{n}=S p\left((Y)_{n-1}\right)$, by using Lemma 18 and an internal induction on $n$ we obtain Fund $\left(\omega_{n}(\omega),(Y)_{0}\right)$.

We can now show that $T$ proves the well-foundedness of ordinals strictly less than $\varepsilon_{\omega}$.

Theorem 20. For each $k<\omega, T \vdash \operatorname{Fund}\left(\varepsilon_{\bar{k}}, X\right)$.

Proof. Since $k$ is given externally, in the formal theory $T$ it is named by $\bar{k}$, the $k$-th numeral. Below, for ease of presentation, we shall identify $k$ and $\bar{k}$. The proof proceeds by external induction on $k$. For a fixed $k$, by internal recursion on $n$ define $\alpha_{0}^{k}=\varepsilon_{k-1}+1$ and $\alpha_{n+1}^{k}=\omega^{\alpha_{n}^{k}}$. This time start with Fund $\left(\alpha_{0}^{k},(Y)_{n}\right)$ and proceed as in Lemma 19 to derive $\forall n$ Fund $\left(\alpha_{n}^{k}, X\right)$. Since $\sup _{n} \alpha_{n}^{k}=\varepsilon_{k}$ we can deduce Fund $\left(\varepsilon_{k}, X\right)$. 


\section{Conclusion}

We have shown that our upper bound for the proof-theoretic ordinal of the theory $T$ is indeed the least one. This allows us to determine the proof-theoretic strength of $T$, and hence that of the infinite Ramsey theorem.

Theorem 21. The theory $\mathrm{ACA}_{0}+\mathrm{iRT}$, i.e. $\mathrm{ACA}_{0}$ augmented by the infinite Ramsey theorem, proves the same arithmetical statements as $\mathrm{PA}+\mathrm{TI}\left(\prec \varepsilon_{\omega}\right)$.

Proof. Since $\mathrm{ACA}_{0}+\mathrm{iRT}$ is equivalent to $\mathrm{ACA}_{0}+\forall n \forall X \exists Y$ jump $(n, X, Y)$, Theorem 20 implies $\mathrm{ACA}_{0}+\mathrm{iRT} \vdash \mathrm{TI}\left(\varepsilon_{\bar{k}}, X\right)$ for every $k<\omega$, and thus $\mathrm{ACA}_{0}+\mathrm{iRT} \vdash$ $\operatorname{TI}\left(\prec \varepsilon_{\omega}\right)$. Corollary 11 provides the other direction.

\section{Acknowledgements.}

The first author was supported by the Engineering and Physical Sciences Research Council UK (grant number EP/G012962/1). The second author was supported by a Royal Society International Joint Projects award 2006/R3.

\section{References}

[1] B. Afshari. Relative computability and the proof-theoretic strength of some theories, Ph.D. Thesis, University of Leeds, U.K., 2008.

[2] H. Friedman, K. McAloon and S. Simpson. A finite combinatorial principle equivalent to the 1-consistency of predicative analysis, In: G. Metakides ed. Patras Logic Symposion, North-Holland, 197-230, 1982.

[3] P.G. Hinman. Recursion-theoretic hierarchies, Springer-Verlag, Berlin, Heidelberg, 1978.

[4] G. Jäger. Theories for iterated jumps, unpublished notes, 1980.

[5] C.G. Jockusch. Ramsey's theorem and recursion theory, Journal of Symbolic Logic 37(1972), 268-280.

[6] K. McAloon. Paris-Harrington incompleteness and progressions of theories, Proceedings of Symposia in Pure Mathematics 42(1985), 447-460.

[7] F.P. Ramsey. On a problem of formal logic, Proceedings of the London Mathematical Society 30(1)(1930), 264-286.

[8] U. Schmerl. A fine structure generated by reflection formulas over primitive recursive arithmetic, In: M. Boffa, K. McAloon, D. van Dalen eds., Studies in Logic and the Foundations of Mathematics, Elsevier 97, 335-350, 1979.

[9] K. Schütte. Proof theory, Springer-Verlag, Berlin, Heidelberg, 1977.

[10] H. Schwichtenberg. Proof theory: Some applications of cut-elimination, In: J. Barwise ed. Handbook of Mathematical Logic, North-Holland, Amsterdam, 867895, 1977.

[11] S.G. Simpson. Subsystems of second order arithmetic, Springer-Verlag, Berlin, Heidelberg, 1999.

[12] M. De Smet and A. Weiermann. A Miniaturisation of Ramsey's Theorem, In: Programs, Proofs, Processes, Proceedings of the 6th international conference on Computability in Europe, LNCS 6158, 118-125, 2010. 